\title{
ELETRODEPOSIÇÃO DE FILMES DE CROMO NEGRO EM SUBSTRATOS DE COBRE PARA ABSORÇÃO SOLAR
}

Ithyara Dheylle Machado de Medeiros ${ }^{1}$, José Felix da Silva Neto ${ }^{2}$, Kerolayne Santos Leite $^{3}$, Aline Karla Barbosa da Silva ${ }^{3}$, Kelly Cristiane Gomes ${ }^{2}$

${ }^{1}$ Doutoranda do Programa de Pós-Graduação em Engenharia Mecânica, UFPB João Pessoa - Brasil, ithyaramachado@gmail.com

${ }^{2}$ Professor(a) Doutor(a) na Universidade Federal da Paraíba, Centro de Energias Alternativas e Renováveis

${ }^{3}$ Mestranda do Programa de Pós-Graduação em Engenharia Mecânica, UFPB

Recebido em: 02/10/2017 - Aprovado em: 21/11/2017 - Publicado em: 05/12/2017 DOI: 10.18677/EnciBio 2017B115

As fontes alternativas e renováveis de energia tais como energia solar, eólica e da biomassa, têm ganhado destaque, inclusive com o crescimento e o fortalecimento de pesquisas envolvendo a utilização dessas formas de energia a nível mundial. A maneira mais simples e direta de aproveitamento da energia solar é através de sua conversão térmica. A conversão solar térmica consiste no aproveitamento do calor transmitido pela radiação solar. Com base nisso, o presente trabalho tem como objetivo avaliar os níveis de absorção de superfícies seletivas absorvedoras de cromo negro depositadas sobre substrato de cobre em função dos parâmetros de eletrodeposição adotados (variação da distância entre os eletrodos e do tempo de deposição), e observar a influência da microestrutura do filme em relação aos níveis de absorção obtidos. Por meio do estudo, foi possível observar que a eletrodeposição de filmes de cromo negro em substratos de cobre proporcionou um aumento de absorção considerável ao longo do espectro em análise (200 - 1400 $\mathrm{nm}$ ), além de estabilidade de absorção. Através das micrografias foi estabelecida uma relação entre a absorção determinada e a microestrutura visualizada, concluindo que o fato dos 4 (quatro) filmes obtidos apresentarem praticamente a mesma absorção está associado com a semelhança microestrutural entre as superfícies.

PALAVRAS-CHAVE: cromo, eletrodeposição, filmes absorvedores.

\section{ELETRODEPOSITION OF BLACK CHROMIUM FILMS IN COPPER SUBSTRATES FOR SOLAR ABSORPTION}

\begin{abstract}
Alternative and renewable sources of energy such as solar, wind and biomass have been highlighted, including the growth and strengthening of research involving the use of these forms of energy worldwide. The simplest and most direct way of harnessing solar energy is through its thermal conversion. Solar thermal conversion consists of taking advantage of the heat transmitted by solar radiation. Based on this,
\end{abstract}


the objective of this work is to evaluate the absorption levels of selective black chromium absorber surfaces deposited on the copper substrate as a function of the adopted electrodeposition parameters (variation of the distance between the electrodes and the time of deposition), and to observe the influence of the microstructure of the film in relation to the absorption levels obtained. Through the study, it was possible to observe that the electrodeposition of black chromium films on copper substrates provided a considerable absorption increase over the spectrum under analysis $(200-1400 \mathrm{~nm})$, besides absorption stability. Through the micrographs, a relationship was established between the determined absorption and the microstructure visualized, concluding that the fact that the 4 films obtained had practically the same absorption is associated with the microstructural similarity between the surfaces.

KEYWORDS: absorber films, chrome, electrodeposition.

\section{INTRODUÇÃO}

Com o desenvolvimento da industrialização, que teve como ponto de partida a Revolução Industrial, o consumo de energia teve um aumento significativo, parcela considerável desse aumento foi em função da utilização da matriz fóssil para obtenção de energia (POMPELLI et al., 2011). Como é de conhecimento comum, essa matriz energética vem sofrendo com o problema de escassez dos seus recursos e dos danos provenientes dos impactos ambientais decorrentes de sua utilização. Dessa forma, as fontes de energias renováveis são uma alternativa de energia limpa e segura, que além de motivar o desenvolvimento tecnológico, proporciona benefícios econômicos indiretos (GOLDEMBERG; LUCON, 2011).

Assim, a diversificação da matriz energética, contemplando o uso das energias renováveis, é uma estratégia valorosa no cenário de crescimento planejado. Dentre essas fontes, destaca-se a energia solar, uma das formas de aproveitamento dessa energia é a conversão solar térmica, na qual a radiação é aproveitada para o aquecimento de um determinado fluido de trabalho (ÇENGEL, 2012). Através de estudos sobre esse tipo de aproveitamento energético, Tabor (1961) e outros autores (SHAFFER, 1958; KOKOROPOULOS et al., 1959) perceberam que alguns materiais poderiam ser projetados e colocados sobre a superfície de outros, de maneira a aumentar o ganho energético com a absorção de radiação e reduzir as perdas energéticas por emissão, aspecto fundamental para a melhoria dos processos de conversão termossolar de energia. Esses revestimentos foram chamados de superfícies seletivas absorvedoras (SSA's).

A idealização desse tipo de revestimento ocorreu para o uso em coletores solares (trocadores de calor utilizados na conversão da energia transferida pela radiação solar em calor). Portanto, fundamentalmente, a seletividade óptica buscada consiste na absorção de toda a radiação emitida pelo sol (na faixa de 300 a 2500 $\mathrm{nm}$ ) e na não-emissão de radiação pelos coletores (TABOR, 1961; CALLISTER Jr.; RETHWISCH, 2016).

A procura pela melhoria da relação absortância/emitância das SSA's tem promovido o estudo de diferentes tipos de revestimentos (ZHENG et al., 2013; KATS et al., 2013; XUE et al., 2013), os filmes seletivos tem se diferenciado através dos seguintes pontos: (i) emprego de materiais que possuem propriedades absorvedoras intrínsecas na faixa espectral emitida pelo sol, (ii) utilização de efeitos de interferência óptica promovida por meio de superposição de revestimentos variados objetivando aumentar a absorção da radiação solar, (iii) produção de armadilhas 
ópticas para a radiação incidente através de texturização de superfícies, (iv) produção de revestimentos a base de compósitos que possibilitam a associação das características de estabilidade térmica dos cerâmicos com a alta condutividade térmica e elétrica dos metais, e ( $v$ ) combinações das demais formas mencionadas acima.

Associada as várias formas existentes de produção da SSA (SANI et al., 2012; JOLY et al., 2013), também é fundamental a escolha da técnica de deposição que será utilizada, visando garantir uma conversão solar térmica homogênea ao longo do coletor, uma boa aderência entre o substrato e o material depositado, e proteção do substrato contra corrosão visto que este é geralmente composto por um material metálico (ATKINSON et al., 2015).

Do ponto de vista comercial, os principais fabricantes de SSA's estão localizados nos Estados Unidos, Europa e Ásia. Esses filmes comerciais podem ser separados em três grupos, em função da temperatura de trabalho do coletor, sendo eles: (i) baixa - até $100^{\circ} \mathrm{C}$; (ii) média - entre $100^{\circ} \mathrm{C}$ e $400^{\circ} \mathrm{C}$; e (iii) alta - acima de $400^{\circ} \mathrm{C}$. A Tabela 1 exibe algumas superfícies seletivas absorvedoras fabricadas comercialmente, destacando suas propriedades ópticas e a faixa de temperaturas na qual elas são estáveis.

TABELA 1. Superfícies seletivas comerciais.

\begin{tabular}{|c|c|c|c|c|c|}
\hline Revestimento & Fabricação & Absortância & $\begin{array}{c}\text { Emissividade } \\
\left(100^{\circ} \mathrm{C}\right) \\
\end{array}$ & $\begin{array}{c}\text { Estabilidade } \\
\text { térmica }\end{array}$ & Fabricante \\
\hline $\begin{array}{l}\text { Aço Inoxidável } \\
\text { modificado }\end{array}$ & $\begin{array}{l}\text { Conversão } \\
\text { química }\end{array}$ & $0,62-0,93$ & 0,10 & $<200^{\circ} \mathrm{C}$ no ar & SEL, INCO \\
\hline $\begin{array}{l}\text { Níquel Negro (NiS- } \\
\text { ZnS) }\end{array}$ & Eletrodeposição & $0,88-0,96$ & $0,03-0,10$ & $<200^{\circ} \mathrm{C}$ no ar & Maxorb \\
\hline $\begin{array}{l}\text { Cromo Negro (Cr- } \\
\left.\qquad \mathrm{Cr}_{2} \mathrm{O}_{3}\right)\end{array}$ & Eletrodeposição & 0,97 & 0,09 & $\begin{array}{l}400^{\circ} \mathrm{C} \text { no vácuo } \\
\text { e } 350^{\circ} \mathrm{C} \text { no ar }\end{array}$ & $\begin{array}{l}\text { MTI, ChromeCoat } \\
\text { Energie Solaire }\end{array}$ \\
\hline $\mathrm{Ni}-\mathrm{NiO}_{x}$ & Sputtering & 0,96 & 0,10 & $300^{\circ} \mathrm{C}$ no ar & Sunstrip \\
\hline $\mathrm{Mo}-\mathrm{Al}_{2} \mathrm{O}_{3}$ & Sputtering & 0,96 & $0,16\left(350^{\circ} \mathrm{C}\right)$ & $\begin{array}{l}350-500^{\circ} \mathrm{C} \text { no } \\
\text { vácuo }\end{array}$ & Solel \\
\hline $\mathrm{W}-\mathrm{Al}_{2} \mathrm{O}_{3}$ & Sputtering & 0,96 & $0,16\left(350^{\circ} \mathrm{C}\right)$ & $\begin{array}{c}350-500^{\circ} \mathrm{C} \text { no } \\
\text { vácuo }\end{array}$ & Solel \\
\hline
\end{tabular}

Fonte: Adaptado de Kennedy (2002) e Selvakumar e Barshilia (2012).

Pela Tabela 1, pode-se observar que dentre os revestimentos disponíveis comercialmente, o cromo negro fabricado por eletrodeposição é o que apresenta melhores características ópticas, além de ser comumente empregado em função da sua simplicidade e baixo custo (DARYABEGY; MAHMOODPOOR, 2006).

Contudo, as pesquisas relativas a eletrodeposição de cromo negro apresentam uma certa limitação em termos do material a ser utilizado como substrato, pois em sua maioria é utilizado aço, porém, é possível encontrar na literatura algumas pesquisas com cobre e níquel como opção de superfície a ser revestida (BAYATI et al., 2005; JAFARI; ROZATI, 2011). No entanto, as análises, aplicadas sobre esses, são bastante restritas, como é o caso do trabalho de Surviliene et al. (2014), que eletrodepositaram cromo negro em substratos de cobre 
e de aço, e só realizaram análises ópticas e microestruturais nos revestimentos fabricados sobre o aço. Já o estudo de Lee (2007), apesar de ter obtido imagens em duas e três dimensões da superfície dos filmes de cromo negro em substratos de cobre, utilizou cobre revestido com níquel.

Dessa forma, o presente trabalho teve como objetivo contribuir para a avaliação dos níveis de absorção de SSA's de cromo negro depositadas sobre substrato de cobre puro em função dos parâmetros de eletrodeposição tempo e distância ânodo-cátodo, e observar a influência da microestrutura do filme em relação aos níveis de absorção obtidos.

\section{Preparo do substrato}

\section{MATERIAL E MÉTODOS}

Antes da eletrodeposição, os substratos de cobre cortados em dimensões de $3 \mathrm{~cm} \times 2 \mathrm{~cm}$ ficaram imersos em éter de petróleo por 30 minutos, sendo posteriormente secados em estufa à $50^{\circ} \mathrm{C} / 1 \mathrm{~h}$.

\section{Eletrodeposição}

A eletrodeposição de cromo negro do presente estudo utilizou um banho composto por $274 \mathrm{~g} / \mathrm{l}$ de trióxido de cromo $\left(\mathrm{CrO}_{3}\right)$ e $0,854 \mathrm{~g} / \mathrm{l}$ de ácido hexafluorossilícico $\left(\mathrm{H}_{2} \mathrm{SiF}_{6}\right)$. A temperatura do banho variou entre 24 e $30^{\circ} \mathrm{C}$, e a densidade de corrente ficou na faixa de 11 a $54 \mathrm{~A} / \mathrm{dm}^{2}$. A variação de temperatura e da densidade de corrente ocorreu pelo fato do regulador de tensão empregado só permitir o ajuste da tensão, enquanto que a corrente oscila em função desta. $O$ ânodo utilizado foi uma liga $\mathrm{Pb}-\mathrm{Sb}$ (95-5\%).

A Figura 1, a seguir, ilustra as dimensões do substrato de cobre bem como as do ânodo empregado, além de exibir as superfícies do filme depositado e do ânodo após a realização das eletrodeposições do presente estudo.

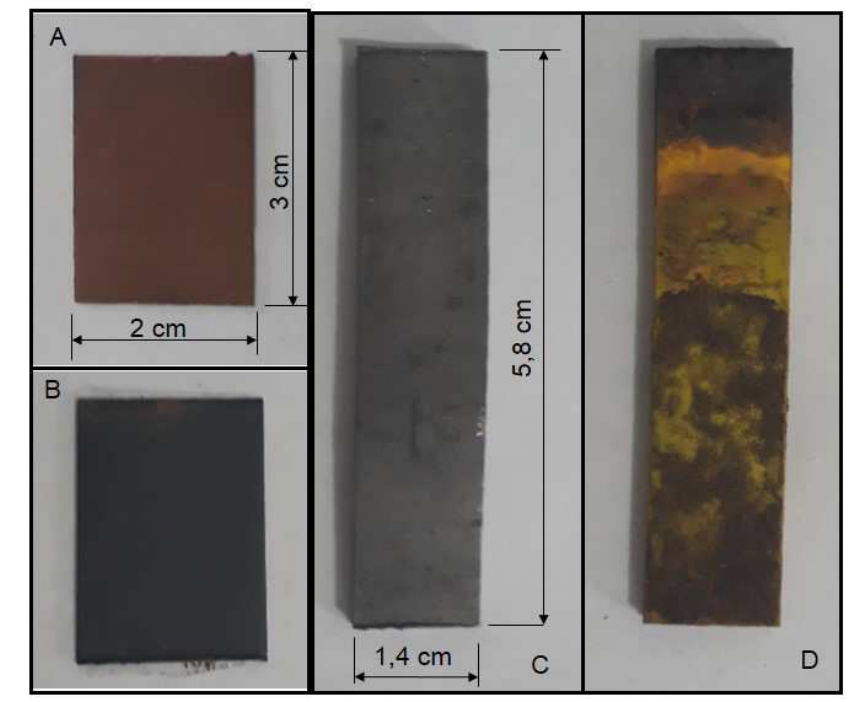

FIGURA 1. A) substrato de cobre antes da eletrodeposição, b) filme eletrodepositado sobre 0 substrato de cobre, c) ânodo de $\mathrm{Pb}-\mathrm{Sb}$ antes das eletrodeposição, d) ânodo após as eletrodeposições. Fonte: Autores (2017). 
2.

Os parâmetros em análise foram variados de acordo com o exposto na Figura

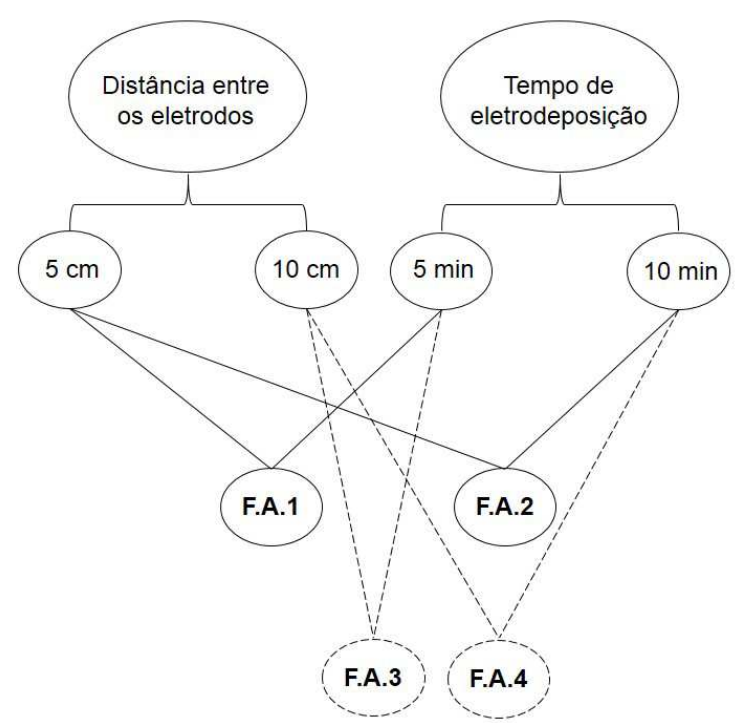

FIGURA 2. Parâmetros de eletrodeposição e nomenclatura dos filmes produzidos. Fonte: Autores (2017).

A eletrodeposição, ilustrada na Figura 3, foi realizada em duplicata de forma que foram utilizados 8 (oito) substratos de cobre, os quais foram divididos em 4 (quatro) condições diferentes e nomeados como Filmes Absorvedores (F.A.), como esquematizado na Figura 2.

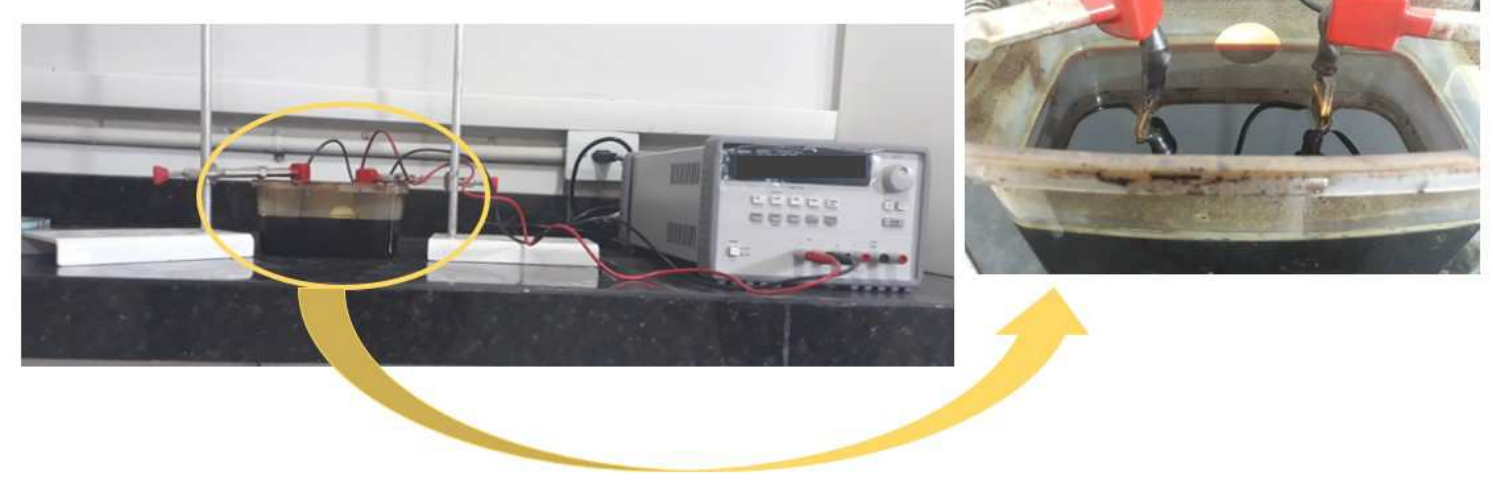

FIGURA 3. Sistema de eletrodeposição com distância ânodo-cátodo de $5 \mathrm{~cm}$. Fonte: Autores (2017).

Os oito substratos de cobre foram submetidos a caracterização óptica antes da eletrodeposição, de forma a poder avaliar o efeito do revestimento sobre esses. A absortância, ao longo do espectro, tanto dos 8 (oito) substratos quanto das duplicatas de cada um dos filmes absorvedores, foi obtida por meio da utilização do Espectrofotômetro na região do Ultravioleta-Visível da marca Shimadzu modelo UV2600 operando na região de 220 a 1400 nm com medidas de absortância, utilizando o acessório esfera de integração, o qual possibilita a análise de filmes.

As amostras passaram também por análise de superfície através de ENCICLOPÉDIA BIOSFERA, Centro Científico Conhecer - Goiânia, v.14 n.26; p.1374 2017 
microscópio eletrônico de varredura com módulo ambiental, marca FEI Modelo Quanta 450, utilizando o princípio de funcionamento de elétrons secundários.

\section{RESULTADOS E DISCUSSÃO}

\section{Espectrofotometria na região do Ultravioleta-Visível (UV-Vis)}

A absortância, ao longo do espectro, do substrato de cobre e dos filmes absorvedores obtidos, está registrada na Figura 4.

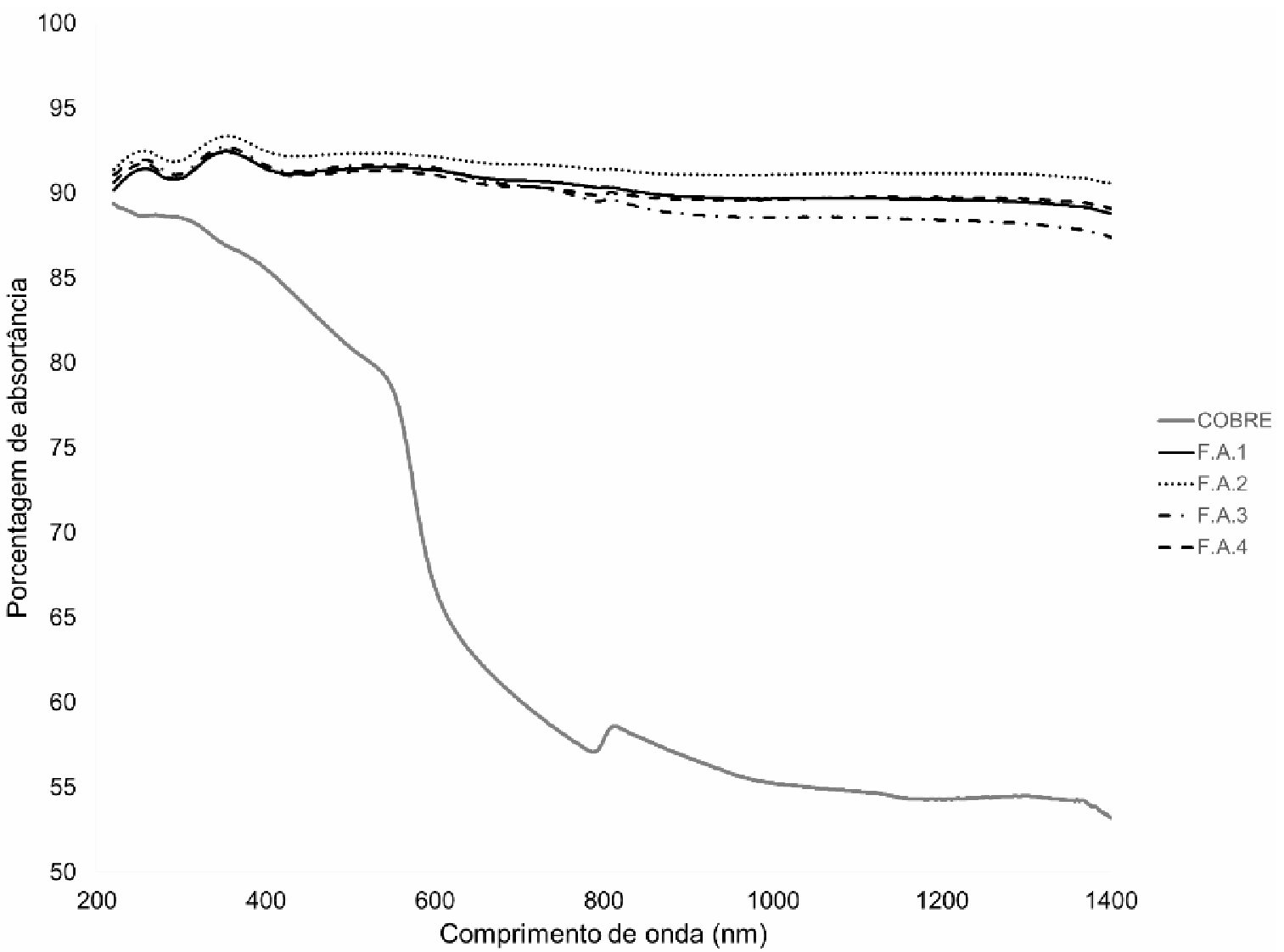

FIGURA 4. Porcentagem de absortância do substrato de cobre e dos filmes absorvedores obtidos por eletrodeposição. Fonte: Autores (2017).

Pela Figura 4, percebe-se que a técnica de eletrodeposição possibilitou a formação de filmes absorvedores estáveis, pois enquanto o nível de absortância no substrato de cobre apresentava uma queda de $40 \%$ entre o início e o fim do espectro em análise, os filmes obtidos mantiveram a absortância.

Já sob o ponto de vista das variáveis estabelecidas para eletrodeposição (distância entre os eletrodos e tempo), a Figura 2 ilustra que independentemente dos parâmetros utilizados, os quatro filmes absorvedores fabricados obtiveram praticamente a mesma resposta em níveis de absorção no espectro em estudo (200 à $1400 \mathrm{~nm}$ ), com destaque para o F.A.2, que além de estável manteve uma absortância acima dos demais. Os resultados obtidos corroboraram com a pesquisa de Bayati et al. (2005), que eletrodepositou durante cinco minutos revestimentos seletivos de cromo negro sobre substratos de cobre e obteve uma média de absortância de 96\%. 


\section{MICROSCOPIA ELETRÔNICA DE VARREDURA (MEV)}

Com o objetivo de analisar a influência da microestrutura superficial dos filmes em relação aos níveis de absortância, foi realizada a análise de MEV. Na Figura 5 pode ser observada as micrografias obtidas.
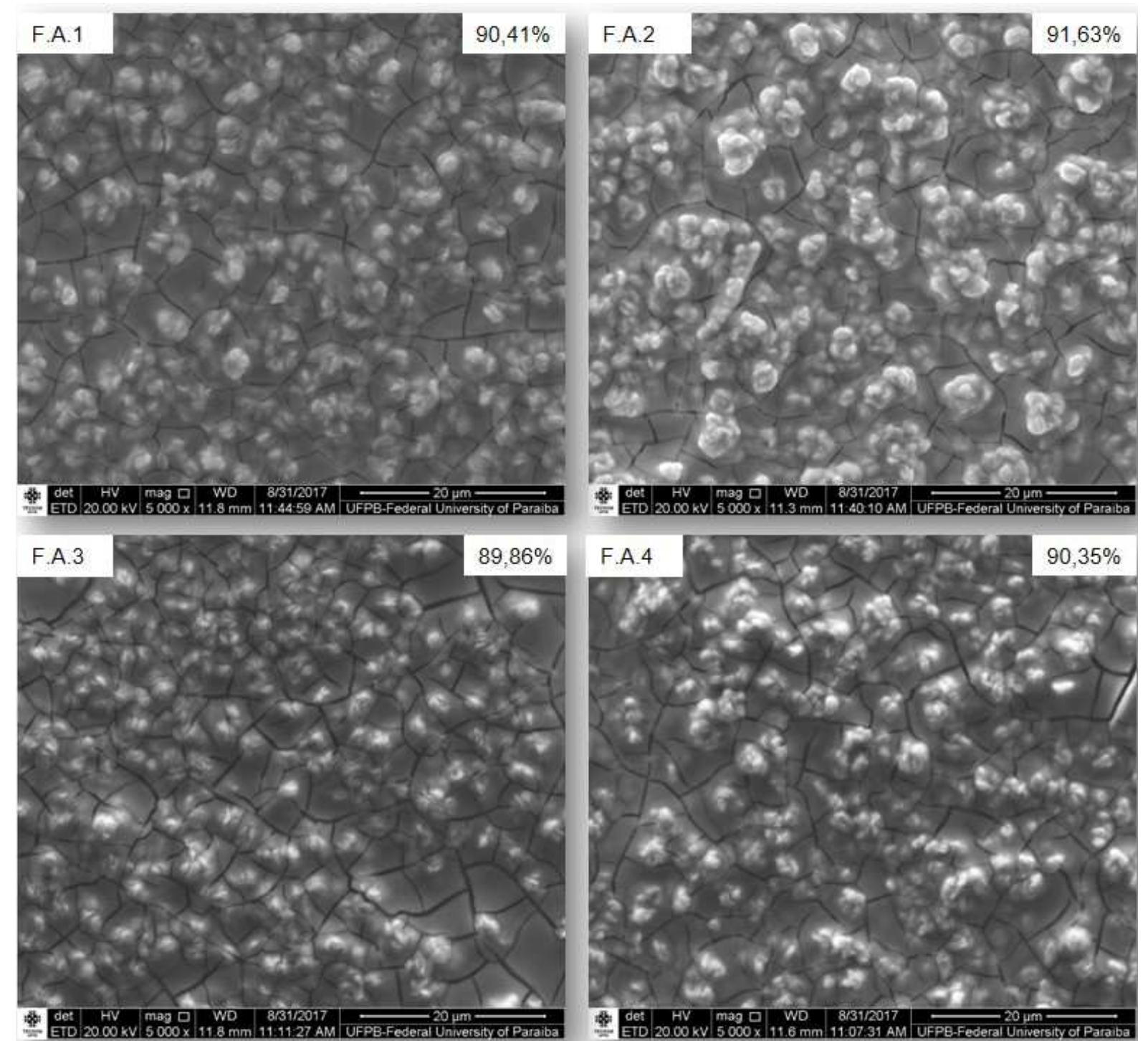

FIGURA 5. Micrografias dos 4 (quatro) Filmes Absorvedores obtidos por eletrodeposição. Fonte: Autores (2017).

Em cada uma das micrografias da Figura 5 está exposta a média geral de absortância obtida por filme. Como discutido anteriormente, as absortâncias dos F.A.'s se mantiveram próximas, isso pode ter sido influenciado pelo fato das superfícies dos filmes apresentarem microestrutura semelhante, conforme pode ser observado (ZHENG et al., 2013; XUE et al., 2013). Percebe-se que o F.A.2 apresenta superfície com maior rugosidade quando comparada com as demais, o que pode ter sido um fator determinante para a sua absortância (GRANQVIST, 2012). 


\section{CONCLUSÃO}

A técnica de eletrodeposição possibilitou a formação de filmes absorvedores estáveis. Independentemente dos parâmetros utilizados, os 4 (quatro) filmes absorvedores fabricados obtiveram praticamente a mesma resposta em níveis de absorção no espectro em estudo (200 à $1400 \mathrm{~nm}$ ), esse fato foi associado a semelhança microestrutural observada nas micrografias das superfícies.

\section{REFERÊNCIAS}

ATKINSON, C.; SANSOM, C.L.; ALMOND, H.J.; SHAW, C.P. Coatings for concentrating solar systems - A review. Renewable and Sustainable Energy Reviews, v. 45, p. 113-122, 2015. Disponível em: < https://doi.org/10.1016/j.rser.2015.01.015>.doi: 10.1016/j.rser.2015.01.015.

BAYATI, M. R.; SHARIAT, M. H.; JANGHORBAN, K. Design of chemical composition and optimum working conditions for trivalent black chromium electroplating bath used for solar termal collectors. Renewable Energy, v. 30, n. 14, p. 2163-2178, 2005. Disponível em: <https://doi.org/10.1016/j.renene.2005.02.003>.doi: 10.1016/j.renene.2005.02.003

CALLISTER JR., W. D.; RETHWISCH, D.G. Ciência e Engenharia dos Materiais Uma Introdução. Editora LTC, 9ª Edição, Rio de Janeiro, 2016.

ÇENGEL, Y.A. Transferência de Calor e Massa - Uma abordagem prática. McGraw Hill, São Paulo, 4를. ed., 2012.

DARYABEGY, M.; MAHMOODPOOR, A. R. Method of manufacturing absorbing layers on copper for solar applications. ISESCO - Science and Technology Vision, v. 2 , n. 1 , p. 35-39, 2006 . Disponível em: < https://www.isesco.org.ma/ISESCO_Technology_Vision/NUM02/doc/08Daryabegy.p df>.

GRANQVIST, G.C. Preparation of thin films and nanostructured coatings for clean tech applications: A primer. Solar Energy Materials \& Solar Cells, v. 99, p.166-175, 2012. Disponível em: <https://doi.org/10.1016/j.solmat.2011.11.048>.doi: 10.1016/j.solmat.2011.11.048.

GOLDEMBERG, José; LUCON, Oswaldo. Energia, Meio Ambiente e Desenvolvimento. São Paulo: Editora da Universidade de São Paulo, 2011.

JAFARI, S.; ROZATI, S. M. Characterization of black chrome films prepared by electroplating technique. In: World Renewable Energy Congress, 2011, Sweden. Disponível em: <http://dx.doi.org/10.3384/ecp110573999>.doi: 10.3384/ecp110573999.

JOLY, M.; ANTONETTI, Y.; PYTHON, M.; GONZALEZ, M.; GASCOU, T.; SCARTEZZINI, J.-L.; SCHULER, A. Novel black selective coating for tubular solar absorbers based on a sol-gel method. Solar Energy, v. 94, p. 233-239, 2013. Disponível em: <https://doi.org/10.1016/j.solener.2013.05.009>.doi: 
10.1016/j.solener.2013.05.009.

KATS, M.A.; BLANCHARD, R.; GENEVET, P.; CAPASSO, F. Nanometre optical coatings based on strong interference effects in highly absorbing media. Nature Materials, v.12, p.20-24, 2013.

KENNEDY, C. E. Review of Mid- to High-Temperature Solar Selective Absorber Materials, NREL/TP-520-31267, National Renewable Energy Laboratory, Colorado, July 2002.

KOKOROPOULOS, P.; SALAM, E.; DANIELS, F. Selective Radiation Coatings. Preparation and high temperature stability. Solar Energy, v. 3, p. 19-23, 1959. Disponível em: < https://doi.org/10.1016/0038-092X(59)90003-9>.doi: 10.1016/0038092X(59)90003-9.

LEE, K. D. Preparation and characterization of black chrome solar selective coatings. Journal of the Korean physical society, v. 51, n. 1, p. 135-144, 2007. Disponível em:< https://doi.org/10.3938/jkps.51.135>.doi: 10.3938/jkps.51.135.

POMPELLI, M. F.; OROZCO, A. J. J.; OLIVEIRA, M. T. de; RODRIGUES, B. R. M.; BARBOSA, M. O.; SANTOS, M. G.; OLIVEIRA, A. F. M. de; ALMEIDA-CORTEZ, J. $S$. de. Crise Energética Mundial e o Papel do Brasil na Problemática de Biocombustíveis. Agronomia Colombiana, Bogotá, v. 29, n. 2, p. 361-371, jun. 2011.

SANI, E.; MERCATELLI, L.; JANFRANCESCO, D.; SANS, J. L.; SCITI, D. Ultra-High Temperature Ceramics for solar receivers: spectral and high-temperature emittance characterization. Journal Of The European Optical Society-Rapid Publications, v. 7, article number 12052, 2012. Disponível em:< https://doi.org/10.2971/jeos.2012.12052>.doi: 10.2971/jeos.2012.12052.

SELVAKUMAR, N.; BARSHILIA, H.C. Review of physical vapor deposited (PVD) spectrally selective coatings for mid- and high-temperature solar thermal applications. Solar Energy Materials \& Solar Cells, v. 98, p. 1-23, 2012. Disponível em: < https://doi.org/10.1016/j.solmat.2011.10.028>.doi: 10.1016/j.solmat.2011.10.028.

SHAFFER, L.H. Wavelength-dependent (selective) processes for the utilization of solar energy. Solar Energy, v. 2, p. 21-26, 1958. Disponível em: < https://doi.org/10.1016/0038-092X(58)90050-1>.doi:10.1016/0038-092X(58)90050-1.

SURVILIENE, S.; CESUNIENE, A.; JUSKENAS, R.; SELSKIENE, A.; BUCINSKIENE, D.; KALINAUSKAS, P.; JUSKEVICIUS, K.; JUREVICIUTE, I. The use of trivalente chromium bath to obtain a solar selective black chromium coating. Applied Surface Science, v. 305, p. 492-497, 2014. Disponível em: < https://doi.org/10.1016/j.apsusc.2014.03.122>.doi: 10.1016/j.apsusc.2014.03.122.

TABOR, H. Solar collectors, selective surfaces and heat engines. Proceedings of the National Academy of Sciences of the United States of America, v. 47, p. 1271-1278, 1961. Disponível em: < http://www.pnas.org/content/47/8/1271.short >. Acesso em: 25 de setembro de 2017. 
XUE, Y.; WANG, C.; WANG, W.; LIU, Y.; WU, Y.; NING, Y.; SUN, Y. Spectral properties and thermal stability of solar selective absorbing $\mathrm{AINi}-\mathrm{Al}_{2} \mathrm{O}_{3}$ cermet coating. Solar Energy, v. 96, p.113-118, 2013. Disponível em: < https://doi.org/10.1016/j.solener.2013.07.012>.doi: 10.1016/j.solener.2013.07.012.

ZHENG, L.; GAO, F.; ZHAO, S.; ZHOU, F.; NSHIMIYIMANA, J.P.; DIAO, X. Optical design and co-sputtering preparation of high performance $\mathrm{Mo}-\mathrm{SiO}_{2}$ cermet solar selective absorbing coating. Applied Surface Science, v. 280, p.240- 246, 2013. Disponível em: <https://doi.org/10.1016/j.apsusc.2013.04.142>.doi: 10.1016/j.apsusc.2013.04.142. 